Proceedings

\title{
Towards Circular Economy: Different Strategies for Polyurethane Waste Recycling and the Obtaining of New Products ${ }^{\dagger}$
}

\author{
Lorena Ugarte 1,** Tamara Calvo-Correas ${ }^{2}$, Itziar Gonzalez-Gurrutxaga ${ }^{3}$, \\ Cristina Peña-Rodriguez ${ }^{2}$, Oihane Etxeberria ${ }^{2}$, Maria Angeles Corcuera ${ }^{2}$ and Arantxa Eceiza ${ }^{2}$ \\ 1 Department of Graphical Expression and Project Management, School of Engineering, Gipuzkoa, \\ Eibar Section, Otaola Hiribidea 29, 20600 Eibar, Spain \\ 2 Department of Chemical and Environmental Engineering, School of Engineering, Gipuzkoa, \\ Plaza Europa 1, 20018 Donostia-San Sebastian, Spain; tamara.calvo@ehu.eus (T.C.-C.); \\ cristina.pr@ehu.eus (C.P.-R.); oihane.etxeberria.95@gmail.com (O.E.); marian.corcuera@ehu.eus (M.A.C.); \\ arantxa.eceiza@ehu.eus (A.E.) \\ 3 Department of Graphical Expression and Project Management, School of Engineering, Gipuzkoa, \\ Plaza Europa 1, 20018 Donostia-San Sebastian, Spain; itziar.gonzalez@ehu.eus \\ * Correspondence: lorena.ugarte@ehu.eus; Tel.: +34-94303-3030 \\ + Presented at the 2nd International Research Conference on Sustainable Energy, Engineering, Materials and \\ Environment (IRCSEEME), Mieres, Spain, 25-27 July 2018.
}

Published: 21 November 2018

\begin{abstract}
As a consequence of the high production and simultaneous consumption of polyurethanes (PU) a great volume of PU waste is landfilled. In this scenario, suitable and efficient routes for PU waste recycling have been searched for many years. In this work two series of PUs using different recycled PU sources were synthesized: a thermoplastic PU series using a glycolysated polyol obtained from the glycolysis of elastomeric PU waste (chemical recycling) and a PU flexible foam series loaded with PU dust waste created in the shaping of PU surf tables (mechanical recycling). Results showed that the incorporation of recycled components in the formulation improved mechanical properties both in the case of thermoplastic polyurethanes and polyurethane foams. The optimum glycolysated polyol was fixed in $15 \%$ over the total polyol weight for thermoplastic PUs. In the case of foams, a maximum of $20 \%$ PU dust over the polyol weight was incorporated.
\end{abstract}

Keywords: polyurethane waste; chemical recycling; mechanical recycling; glycolysis; polyol; thermoplastic polyurethanes; polyurethane foams

\section{Introduction}

Global demand of polyurethane (PU) products reached 15 million tons in 2013 and it is expected to reach 22 million tons in 2020. As a consequence of the high production and simultaneous consumption of these materials, a great volume of PU waste is landfilled. In this scenario, suitable and efficient routes for PU waste recycling have been searched for many years. Recycling techniques can be classified as primary, secondary, tertiary and quaternary. Primary and secondary recycling, gathered as mechanical recycling, consist on the direct reuse of uncontaminated discarded polymer into a new product. Tertiary recycling or chemical recycling consists on the depolymerization of PU to obtain monomers for their further re-polymerization to regenerate new PU. Finally, in quaternary recycling the material is incinerated in order to recover energy. Concerning chemical recycling of polyurethanes, glycolysis is one of the most studied techniques [1]. It consists on a transesterification reaction implying an exchange of the ester group of the urethane by the hydroxyl group of a glycol, 
obtaining a recycled product that can replace the polyol component. The obtained polyol can be used in the synthesis of new polyurethane foams [2] or elastomers [3] and it constitutes the soft segments (SS) of the polyurethane conferring ductility, extensibility and recovering ability to the polyurethane. Urethane rich segments constitute the hard segments (HS) of the material, acting as reinforcement and complementing the microstructure of polyurethanes.

The objective of this work was to synthesize two series of PUs using different recycled PU sources: a thermoplastic PU series using a glycolysated polyol obtained from the glycolysis of elastomeric PU waste (chemical recycling) [4] and a PU flexible foam series loaded with PU dust waste created in the shaping of PU surf tables (mechanical recycling). The effect of the added amounts of glycolysated polyol and PU dust on the final properties of the thermoplastic PU elastomers and flexible PU foams was studied, respectively, in order to establish the optimum recycled PU waste content in each series.

\section{Materials and Methods}

For the synthesis of thermoplastic polyurethanes, poly(ethylene-butylene adipate) diol (PEBA) was used as synthetic polyol (Poliols 55/10, Purinova, Chicago, IL, USA) (54.45 mg KOH g-1 $2060 \mathrm{~g}$ mol $^{-1}$ ). 4,4'-Diphenylmethane diisocyanate (MDI) (Desmodur 44, kindly supplied by Covestro, Leverkusen, Germany) and 1,4-butanediol (BD) (Aldrich, St. Louis, MO, USA) were used as diisocyanate and chain extender, respectively. For the synthesis of flexible polyurethane foams a castor oil based polyol (Lupranol Balance ${ }^{\circledR}$ 50, BASF, Ludwigshafen, Germany) (49.7 mg KOH g ${ }^{-1}$, $3048 \mathrm{~g} \mathrm{~mol}^{-1}$ ), water as blowing agent, and toluene diisocyanate (TDI, kindly supplied by Covestro, Leverkusen, Germany) were used, together with catalysts and surfactants.

Elastomeric PU waste was recycled by glycolysis process. Ethylene glycol (EG) (POCH) was used as glycolysis agent and potassium acetate (KAc) (POCH) as catalyst. 10:1 wt (PU waste:EG), $0.5: 100 \mathrm{wt}$ (KAc:PU) ratios were used for the reaction, carried out at $180-190^{\circ} \mathrm{C}$ for $40 \mathrm{~min}$. A viscous polyol was obtained (Gly) (199 $\left.\mathrm{mg} \mathrm{KOH} \mathrm{g}^{-1}, 564 \mathrm{~g} \mathrm{~mol}^{-1}\right)$. Thermoplastic polyurethanes were obtained by two step bulk polymerization. On the first step PEBA, Gly and MDI were reacted for $5 \mathrm{~h}$ at $80^{\circ} \mathrm{C}$. On the second step, BD chain extender was added at $60^{\circ} \mathrm{C}$ and the mixture was vigorously agitated for $10 \mathrm{~min}$. The obtained viscous liquid was poured between two Teflon ${ }^{\circledR}$ coated metal sheets and was left to cure for $10 \mathrm{~h}$, at $180{ }^{\circ} \mathrm{C}$ and 50 Bar. NCO:OH ratio was kept constant at 1.05 in all formulations. Samples were designated as PU-XGly, were X indicates the $\mathrm{wt} \%$ of Gly over total polyol weight. Designation, PEBA:Gly:MDI:BD molar ratio and HS content (wt\%) of each sample are shown in Table 1.

Table 1. Designation, reactants molar ratio and HS content (wt\%) of synthesized thermoplastic polyurethanes.

\begin{tabular}{ccccc}
\hline Sample & PEBA:Gly & MDI & BD & HS content (wt\%) \\
\hline PU-0Gly & $1: 0$ & 3.15 & 2 & 32 \\
PU-5Gly & $0.85: 0.15$ & 3.15 & 2 & 35 \\
PU-10Gly & $0.7: 0.30$ & 3.15 & 2 & 38 \\
PU-15Gly & $0.6: 0.4$ & 3.15 & 2 & 40 \\
PU-20Gly & $0.5: 0.5$ & 3.15 & 2 & 42 \\
PU-25Gly & $0.45: 0.55$ & 3.15 & 2 & 44 \\
\hline
\end{tabular}

Foams were synthesized at room temperature. Polyol, PU dust waste, catalysts ( 0.35 parts per hundred polyol, pphp), water (3.5 pphp) and surfactant (1.1 pphp) were mixed at $2000 \mathrm{rpm}$ for $2 \mathrm{~min}$. Then, the fixed amount of TDI (Isocyanate Index $=110$ ) was incorporated and mixing continued for $10 \mathrm{~s}$ at the same speed. The mixture was then quickly poured into an open mold to left the foam rise freely. Foams were cured at room temperature for at least $24 \mathrm{~h}$ before characterization. The formulation was kept constant and PU dust was incorporated to the polyol in different amounts. Foams were designated as PUF-X where $X$ denotes the $\mathrm{w} t \%$ of PU dust over the polyol weight. Foam designation, density and obtained compressive mechanical properties are gathered in Table 2. 
Table 2. Designation and density of obtained flexible polyurethane foams.

\begin{tabular}{cc}
\hline Sample & Density $\left.\mathbf{~ k g ~ m}^{-3}\right)$ \\
\hline PUF-0 & $37.4 \pm 0.5$ \\
PUF-10 & $47.2 \pm 0.2$ \\
PUF-15 & $49.7 \pm 0.6$ \\
PUF-20 & $51.2 \pm 1.3$ \\
\hline
\end{tabular}

\section{Results and Discussion}

Fourier transformed infrared spectroscopy (FTIR) results of PEBA, Gly and synthesized thermoplastic polyurethanes are shown in Figure 1a. When comparing the spectra of PEBA and Gly, the presence of $\mathrm{OH}$ groups was identified at around $3500 \mathrm{~cm}^{-1}$ in both polyols. Besides, the presence of N-H $\left(3363 \mathrm{~cm}^{-1}\right), \mathrm{C}-\mathrm{N}\left(1306 \mathrm{~cm}^{-1}\right)$ and C-O $\left(1220 \mathrm{~cm}^{-1}\right)$ groups was evidenced in the spectrum of Gly polyol. This suggested that some residual urethane groups remained on Gly polyol after the glycolysis process. Analyzing the spectra of thermoplastic polyurethanes, there was no evidence of unreacted NCO groups $\left(2270 \mathrm{~cm}^{-1}\right)$. All samples showed typical polyurethane spectrum with peaks corresponding to $\mathrm{N}-\mathrm{H}\left(3334 \mathrm{~cm}^{-1}\right), \mathrm{C}=\mathrm{O}\left(1730 \mathrm{~cm}^{-1}\right), \mathrm{C}-\mathrm{N}\left(1306 \mathrm{~cm}^{-1}\right)$ and $\mathrm{C}-\mathrm{O}\left(1220 \mathrm{~cm}^{-1}\right)$ groups.

Mechanical properties of samples were tested in tensile mode and the obtained stress-strain curves are shown in Figure 1b. Young's Modulus increased in proportion to Gly content, presumably due to the increase in HS content of the samples and the residual urethane groups. Tensile strength and elongation at break values showed a maximum at $15 \mathrm{wt} \%$ Gly content and then decreased. This behavior suggested that at high Gly contents phase separation was hindered, probably due to the increase in heterogeneity of polyol mixture. In fact, while Young's modulus is related with HS content [5], tensile strength and elongation at break values seem more susceptible to the degree of phase separation between hard and soft segments.
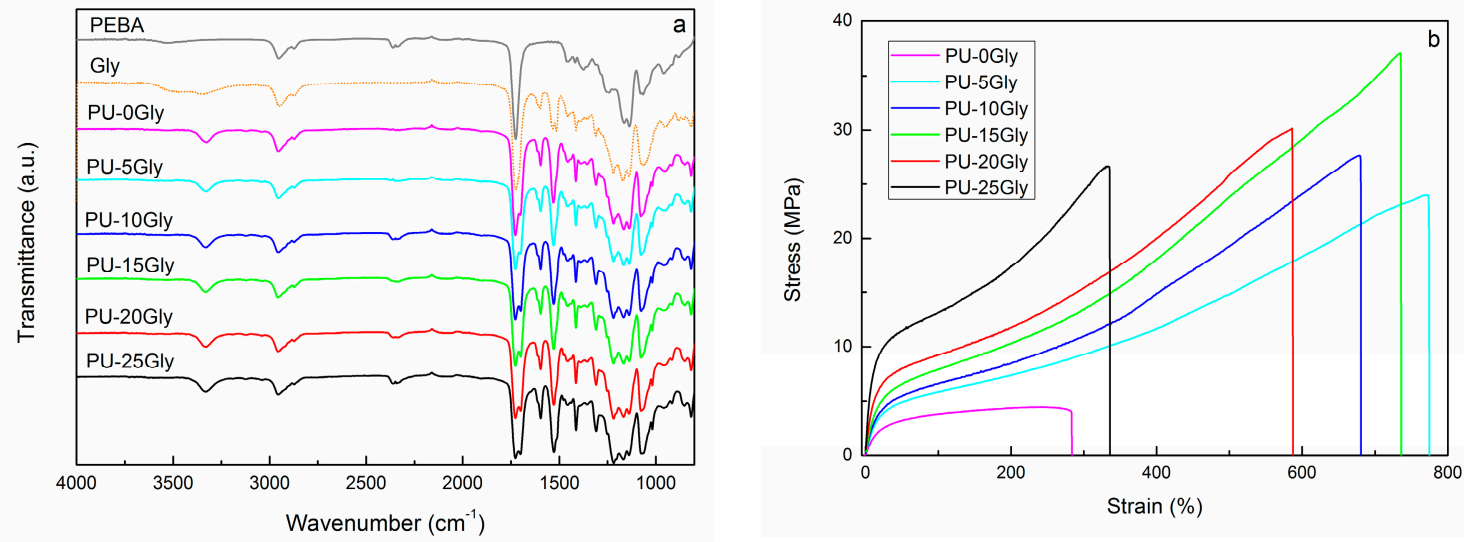

Figure 1. (a) FTIR spectra of Gly, PEBA and synthesized thermoplastic polyurethanes, and (b) tensile stress-strain curves of synthesized thermoplastic polyurethanes.

Concerning polyurethane foams, it was observed that the density of the samples increased as PU dust content increased (Table 2). Moreover, as it can be observed in Figure 2, an important improvement of compressive mechanical properties was achieved with PU dust incorporation. Specifically, compressive strength and compressive modulus values increased more than $100 \%$ and $200 \%$, respectively. Resilience values also improved with PU dust incorporation. 


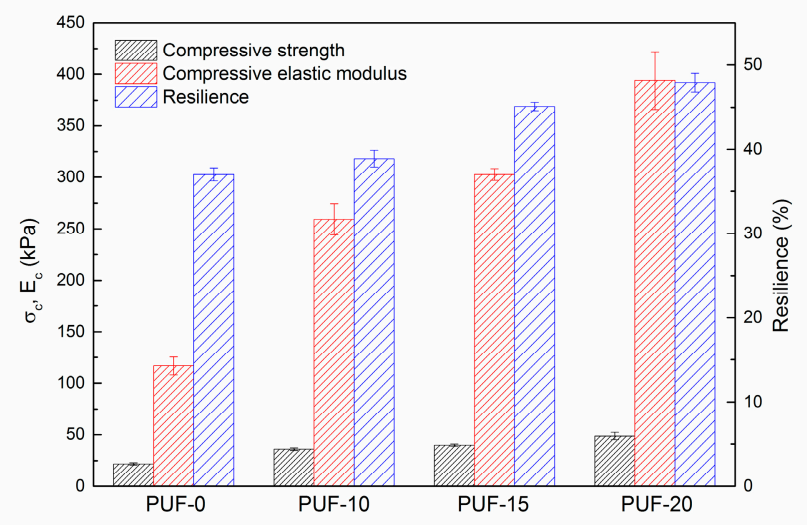

Figure 2. Compressive strength, compressive modulus and resilience values of polyurethane foams.

\section{Conclusions}

Both mechanical and chemical recycling processes showed to be effective for the management of polyurethane waste. New polyurethane products, thermoplastic and foams, with improved mechanical properties were successfully obtained from the recycling of different polyurethanes waste. In the case of thermoplastic polyurethanes, the optimum glycolysated polyol content was fixed at $15 \mathrm{wt} \%$ over the total polyol weight. For polyurethane foams, the maximum polyurethane waste dust content was fixed at $20 \mathrm{wt} \%$ over polyol weight due to limitations in the foaming process.

Author Contributions: L.U. analyzed the data and wrote the paper. T.C.-C. and O.E. performed the experiments, T.C.-C. also analyzed the data. C.P.-R., M.A.C and A.E. conceived and designed the experiments. I.G.-G. contributed to write the paper.

Acknowledgments: We thank the Basque Government (IT-776-13) for the financial support. We also thank for technical and human support provided by SGIker of UPV/EHU and European funding (ERDF and ESF), and Olatu S.A (Gipuzkoa) for providing the PU dust. T.C.-C. thanks the University of the Basque Country (DOCREC17/13) and Provincial Country of Gipuzkoa (2017-BE01-000002-01).

Conflicts of Interest: The authors declare no conflict of interest.

\section{References}

1. Simón, D.; Borreguero, A.M.; de Lucas, A.; Rodriguez, J.F. Recycling polyurethanes from laboratory to industry, a journey towards de sustainability. Waste Manag. 2018, 76, 147-171, doi:10.1016/j.wasman.2018.03.041.

2. Nikje, A.; Mohammad, M.; Haghshenas, M.; Garmarudi, A.B. "Split-phase" glycolysis of flexible PUF wastes and application of recovered phases in rigid and flexible foams production. Polym. Plast. Technol. Eng. 2007, 46, 265-271, doi:10.1080/03602550601153091.

3. Kopczynska, P.; Calvo-Correas, T.; Eceiza, A.; Datta, J. Synthesis and characterization of polyurethane elastomers with semi-products obtained from polyurethane recycling. Eur. Polym. J. 2016, 85, 26-37, doi:10.1016/j.eurpolymj.2016.09.063.

4. Calvo-Correas T.; Ugarte, L.; Jutrzenka Trzebiatowska, P.; Rafael S., Datta J., Corcueraa M., Eceiza A. Thermoplastic polyurethanes with glycolysate indetermediates from polyurethane waste rrecycling. Poym. Degrad. Stab. 2017, 144, 411-419, doi:10.1016/j.polymdegradsTable 2017.09.001.

5. Auad, M.L.; Mosiewicki, M.A.; Richardson, T.; Aranguren, M.I.; Marcovich, N.E. Nanocomposites made from cellulose nanocrystals and tailored segmented polyurethanes. J. Appl. Polym. Sci. 2010, 1215-1225, doi:10.1002/app.31218. 